J. Korean Math. Soc. 48 (2011), No. 6, pp. 1327-1328

http://dx.doi.org/10.4134/JKMS.2011.48.6.1327

\title{
ERRATUM TO "ON LORENTZIAN QUASI-EINSTEIN MANIFOLDS, J. KOREAN MATH. SOC. 48 (2011), NO. 4, PP. 669-689"
}

Absos Ali Shaikh, Young Ho Kim, and Shyamal Kumar Hui

In page 670 , some terms are missing. Also in page 675 , Theorem 3.2 must undergo some changes. In pages $686-687$, some portions will be deleted. In page 688 some additional references will be included. The changes are the following:

Page 670, Line 12:

Should read as: Such a non-Einstein quasi-Einstein manifold of dimension $n \geq$ 3 is denoted by $Q E_{n}$. We note that there exist non-Einstein quasi-Einstein manifolds $(M, g)$ such that the vector $\rho$ is a null vector, i.e., $g(\rho, \rho)=0$ holds on $U_{S} \subset M$, e.g. see Example 5.1 of [2].

Page 670, Line 19:

Should read as: Also three dimensional Cartan hypersurfaces are non-Einstein quasi-Einstein manifolds [3].

Page 670 , Line-11 to line-9:

Should read as: A Lorentzian quasi-Einstein manifold (briefly, $L Q E_{n}$ ) is a non-Einstein quasi-Einstein manifold with the generator $\rho$ as the unit timelike vector field such that $g(\rho, \rho)=-1$. Thus a $L Q E_{n}$ is a non-Einstein Lorentzian manifold whose Ricci tensor satisfies (1.1) such that the generator $\rho$ is the unit timelike vector field. Hence, a $Q E_{n}$ with a Lorentzian metric is a $L Q E_{n}$.

Page 675, Theorem 3.2:

Theorem 3.2. Let $\left(M^{n}, g\right), n \geq 3$, be a connected orientable Lorentzian manifold which is either non-compact or compact with vanishing Euler number. If there is a unit timelike generic vector field $\rho$ on $M$ such that the relation $S \wedge S=2 \alpha g \wedge S+2 \beta G$ holds with $\alpha^{2}+\beta=0$, then the manifold is a $L Q E_{n}$.

In Section 6 (pages 686-687) from Example 6.5 upto the statement of Theorem 6.1 should be deleted.

Received June 28, 2011.

2010 Mathematics Subject Classification. 53B30, 53B50, 53C50, 53C80, 83D05.

Key words and phrases. quasi-Einstein manifold, Lorentzian quasi-Einstein manifold. 
Page 688, the following references should be added:

\section{References}

[1] U. C. De and B. K. De, On quasi Einstein manifolds, Commun. Korean Math. Soc. 23 (2008), no. 3, 413-420.

[2] R. Deszcz and M. Hotloś, On hypersurfaces with type number two in space forms, Annales Univ. Sci. Budapest. Eötvös Sect. Math. 46 (2003), 19-34.

[3] R. Deszcz, L. Verstraelen, and S. Yaprak, Pseudosymmetric hypersurfaces in 4dimensional spaces of constant curvature, Bull. Inst. Math. Acad. Sinica 22 (1994), no. $2,167-179$

Absos Ali Shaikh

Department of Mathematics

UNIVERSITY OF BURDWAN

BURDWAN - 713104

West Bengal, India

E-mail address: aask2003@yahoo.co.in

Young Ho KIM

Department of Mathematics

Teachers College

Kyungpook National University

TAegu 702-701, Korea

E-mail address: yhkim@knu.ac.kr

Shyamal Kumar Hui

Department of Mathematics

UNIVERSITY OF BURDWAN

BURDWAN - 713104

West BengAL, INDIA 